\title{
Sustained alpha-sarcoglycan gene expression following gene transfer in LGMD2D
}

\author{
Jerry R. Mendell ${ }^{1,2,4}$, Louise R. Rodino-Klapac ${ }^{1,4}$, Xiomara Q. Rosales ${ }^{4}$, Brian D. Coley ${ }^{3}$, \\ Gloria Galloway ${ }^{1,2,4}$, Sarah Lewis ${ }^{4}$, Vinod Malik ${ }^{4}$, Chris Shilling ${ }^{4}$, Barry J. Byrne ${ }^{6,7}$, Thomas \\ Conlon $^{6,7}$, Katherine J. Campbell ${ }^{5}$, William G. Bremer ${ }^{5}$, Laura E. Taylor ${ }^{4}$, Kevin M. \\ Flanigan $^{1,4}$, Janaiah Kota ${ }^{4}$, Zarife Sahenk ${ }^{1,2,4}$, Christopher M. Walker ${ }^{1,5}$, and K. Reed \\ Clark $^{1,4}$ \\ ${ }^{1}$ Department of Pediatrics, Ohio State University, Columbus, $\mathrm{OH}$ \\ 2Department of Neurology, Ohio State University, Columbus, $\mathrm{OH}$ \\ ${ }^{3}$ Department of Radiology Ohio State University, Columbus, $\mathrm{OH}$ \\ ${ }^{4}$ Center for Gene Therapy The Research Institute at Nationwide Children's Hospital, Columbus, \\ $\mathrm{OH}$ \\ ${ }^{5}$ Center for Vaccines and Immunity The Research Institute at Nationwide Children's Hospital, \\ Columbus, $\mathrm{OH}$ \\ ${ }^{6}$ Department of Pediatrics, University of Florida College of Medicine, Gainesville,FL \\ ${ }^{7}$ Powell Gene Therapy Center, Gainesville, FL
}

\section{Abstract}

Objective-The aim of this study was to attain long-lasting alpha-sarcoglycan gene expression in LGMD2D subjects mediated by adeno-associated virus (AAV) gene transfer under control of a muscle specific promoter (tMCK)

Methods-rAAV1.tMCK.hSGCA $\left(3.25 \times 10^{11} \mathrm{vg}\right)$ was delivered to the extensor digitorum brevis (EDB) muscle of three subjects with documented SGCA mutations via a double-blind, randomized, placebo controlled trial. Control sides received saline. The blind was not broken until the study was completed at 6 months and all results reported to the oversight committee.

Results-Persistent alpha-sarcoglycan gene expression was achieved for six months in two of three LGMD2D subjects. Markers for muscle fiber transduction other than alpha-sarcoglycan included expression of major histocompatability complex I (MHC I), increase in muscle fiber size, and restoration of the full sarcoglycan complex. Mononuclear inflammatory cells recruited to the site of gene transfer appeared to undergo programmed cell death demonstrated by TUNEL and caspase-3 staining. A patient failing gene transfer demonstrated an early rise in neutralizing antibody titers and T cell immunity to AAV validated by enzyme-linked immunospot (ELISpot) on the second day post gene injection. This was in clear distinction to other participants with satisfactory gene expression.

Interpretation-The findings of this gene replacement study in LGMD2D subjects have important implications not previously demonstrated in muscular dystrophy. Long-term, sustainable gene expression of alpha-sarcoglycan was observed following gene transfer mediated

Address correspondence to: Jerry.Mendell@ nationwidechildrens.org (this can be published)..

This study has been registered at Clinicaltrials.gov NCT00494195.

There are no contributors to this manuscript who have any conflict of interest 
by AAV. The merit of a muscle specific tMCK promoter, not previously used in clinical trial was evident, and the potential for reversal of disease was displayed.

\section{Introduction}

Successful gene therapy for muscle disease will require sustained gene expression using tissue specific regulatory elements resulting in therapeutic proteins expressed at sufficient levels to improve function ${ }^{1}$. In this early phase in the evolution of gene therapy for muscle disease it would be advantageous to assess gene expression at the site of gene transfer in a single patient at sequential time points. This would require consecutive biopsies of the same muscle over a designated time period. This multiple biopsy regimen is clinically impractical and subject to reduced expression levels as vector genomes are lost from the site of gene transfer by prior removal of transduced muscle fibers. In lieu of this approach and in order to maximize interpretation, we have examined gene expression levels at different time points in six different LGMD2D, alpha-sarcoglycan deficient patients receiving intramuscular gene transfer of the full length alpha-sarcoglycan $(\alpha \mathrm{SG}) \mathrm{cDNA}$ under control of a truncated muscle creatine kinase promoter (tMCK). ${ }^{3,4}$ In three patients previously reported ${ }^{2}$, post gene transfer muscle biopsies were examined between 6 weeks and 3 months. This report extends the period of observation post gene transfer for six months in three additional patients and tests the capacity of the tMCK promoter to sustain long-term gene expression. There is currently no effective treatment of LGMD2D, a disease that varies in severity depending on levels of $\alpha \mathrm{SG}$ protein expression; at one end of the spectrum patients suffer from ambulatory loss between 12-15 years of age, while others maintain walking until the third to fourth decade 5,6 .

It would be misleading to present the gene transfer findings of the first three of six LGMD2D patients as new information in this report since the results of these cases have been published ${ }^{7}$. However, summarizing the data from these patients does provide an overall perspective by which to appreciate the findings of the entire cohort. In the first three LGMD2D cases the full length human $\alpha$-SG gene (hSGCA) was transferred to the extensor digitorum brevis (EDB) muscle using ultrasound guidance and electromyographic (EMG) monitoring. The hSGCA dose was $3.25 \times 10^{11}$ vector genomes $(\mathrm{vg})$ delivered in $1.5 \mathrm{ml}$. Two patients (cases 1 and 3 ) had follow up muscle biopsies at 6 weeks and the third underwent muscle biopsy at 3 months. Analysis (performed blinded to side of gene transfer) revealed transduction of $57 \%$ of fibers in subject $1,69 \%$ in subject 2 , and $62 \%$ in subject 3 . Sections from these same blocks taken for western blots showed a 4 to 5 fold increase in all three cases. Two signs of muscle repair included restoration of the full sarcoglycan complex on the side of gene transfer in all cases and an increase in muscle fiber size compared to the control side receiving only vector diluent in one case (subject 3 ). A potentially important finding in all three cases was the expression of MHC class I molecules on the sarcolemma of virtually every muscle fiber on the side of upregulated gene expression, contrasting sharply with the lack of expression on the control side. MHC class II expression was not observed. CD4 and CD8 positive infiltrates tended to be focal (so total numbers were not significantly increased) but suggested recruitment to the side of gene transfer. IFN- $\gamma$ ELISpots showed a minimal but definite response to a specific AAV capsid pool in one of three cases at days 14 and 43 suggesting a transient $T$ cell mediated immune response to capsid pool 2 of AAV1. There was no evidence of an immune response to newly expressed $\alpha$-SG peptides.

The results of the first three cases provided a template upon which to move forward. Although, dose escalation had been originally proposed in the IND, the findings in the first cohort precluded any need to change the regimen and the final three cases received the same amount of vector, to the same muscle, providing an unequivocal basis for comparison at a later timepoint. The fundamental question to be addressed in cohort 2 was whether gene 
expression could be maintained at a robust level for an additional 3 months (total of 6 months) using the tMCK promoter. Additionally we sought to ascertain the potential consequences of universally expressed MHC class I on virtually every muscle fiber on the side of gene transfer and whether a focal cellular inflammatory infiltrate was poised to attack transduced muscle fibers. Perhaps a somewhat more difficult question to address, but one of interest would be the increase in muscle fiber size at the 3-month time point and whether this was a chance occurrence or a result of sustained gene expression.

\section{MATERIALS AND METHODS}

\section{Study Subjects}

Subject eligibility included proof of $S G C A$ mutations of both alleles, ability to cooperate for testing, willingness to practice contraception during the study (if appropriate), negative pregnancy test (for females), and no evidence of cardiomyopathy, diabetes, or organ system abnormalities of bone marrow, liver, or kidney. Human immunodeficiency virus infection, hepatitis A, B, or C, or known autoimmune diseases were exclusion criteria. IRB approved consent forms were obtained by the principal investigator (JRM) and signed by subjects. Taking immunosuppressive drugs or glucocorticoids during the trial was prohibited, and patients were required to be off treatment for 3 months prior to enrollment.

\section{Novel SGCA mutations}

Variations within the $S G C A$ coding sequence were detected in all six patients by direct sequencing analysis of the entire coding region. In two of the patients, previously unreported variants were described. The results of our studies suggest probable pathogenicity of these mutations.

Subject 6-The c.434C>A mutation results in an amino acid substitution at a highly conserved residue (p.A145E), and ablates an AciI restriction endonuclease site. Pathogenicity was assessed by reducing the possibility that this was a rare polymorphism rather than a disease-causing allele. DNA from the patient and from 96 wild-type controls (CEPH parent sets) were PCR amplified using a forward primer in exon 5 (5'-

CCCTGCTGCCATACCAAG-3') and a reverse primer at the junction of exon 5 and intron 5 (5'-GCACACCTACCCTTCTTTTCG-3') resulting in a 206 bp fragment. Restriction digest was performed with AciI (New England Biolabs, MA, USA) under the following conditions: $6.5 \mu \mathrm{L} \mathrm{H} \mathrm{H}_{2} \mathrm{O}, 1.5 \mu \mathrm{L} 10 \times$ NEBuffer $3,1 \mu \mathrm{L}$ restriction enzyme, $6 \mu \mathrm{L}$ PCR product. Samples were incubated at $37^{\circ} \mathrm{C}$ for 1 hour and heat inactivated at $65^{\circ} \mathrm{C}$ for 20 minutes. Expected fragment sizes were 129 and $77 \mathrm{bp}$ for the patient allele and 129, 45, and $32 \mathrm{bp}$ for the wildtype allele. Digestion fragments were analyzed via gel electrophoresis on a $4 \%$ GenePure Sieve agarose (ISC Bioexpress, UT, USA) in $1 \times$ TBE stained with ethidium bromide. Consistent with pathogenicity, the c.434C>A allele was detected only in the patient and not in the 192 control chromosomes.

Subject 5-Sequencing from lymphocyte-derived genomic DNA samples in each parent of patient 5 revealed that each carried one of the putative mutations. Using an archived muscle biopsy specimen from patient, mRNA was extracted from forty 10-micron sections using Trizol (Invitrogen) according to the manufactures recommended protocol. In order to assess the splicing implications of the c.956+2_956+19del mutation, gene-specific rtPCR was performed using SuperScript ${ }^{\circledR}$ One-step RT-PCR System with Platinum ${ }^{\circledR}$ Taq DNA Polymerase (Invitrogen), with a forward primer within exon 6 (5' -

GCACCCCACTTCCGCGTTGAC -3') and a reverse primer within exon 9 (5'GAATGAGGGGCACCTGGGCG -3'). The 5'UTR deletion detected in genomic DNA was confirmed by gene-specific rt-PCR using a forward primer 5' of the canonical UTR (5'- 
AGGTGACTGGAAGGTTGCTG -3') and a reverse primer within the coding region of exon 1 (5'- CAGAAGAGGGATCTGGGTTG -3'). In each case, amplicons were gel-purified and TA cloned into the $\mathrm{pCR} \otimes 2.1 \mathrm{TOPO}{ }^{\circledR}$ vector; clones were subsequently sequenced into using $\mathrm{ABI}$ dye terminator sequencing technologies on an $\mathrm{ABI} 3130 \mathrm{xl}$ sequencer.

With amplification of exons 6 through 9, several species of mRNA were detected that confirm the pathogenicity of the c.956+2_956+19del mutation. Among the two most abundant, one contained a deletion of exon 7, resulting in an out-of-frame transcript (r. 748_956del), whereas the second contained the last 79 nucleotides from intron 6 and the first 46 nucleotides from exon 7 as a pseudoexon between exons 6 and 8 (r.

747_957ins748-79_793). The c.-205_-37del, in contrast, ablates most of the SGCA promoter (including the putative transcriptional start site at c.-175) as predicted by neural network algortithms ${ }^{8}$ and is thus predicted to result in a null allele.

\section{Immune responses}

Subjects were pre-screened for serum neutralizing antibodies to AAV1 according to a previously published protocol ${ }^{9}$, with follow up titers on post gene transfer days $2,7,14,28$, $42,60,90,120,150$, and 180. In addition, an AAV particle binding ELISA assay, similar to that previously published by our group ${ }^{9}$, used serum obtained pre-treatment coinciding with neutralizing antibody titers. For this assay the AAV1 antigen coating protocol was modified to use $2 \times 10^{9} \mathrm{vg}$ particles per 96 well $\left(100 \mu \mathrm{l}\right.$ added per well of $2 \times 10^{10} \mathrm{AAV} 1 \mathrm{particles} / \mathrm{ml}$ in carbonate coating buffer). Pre-screening and follow up studies to identify potential T cell immunity were done on these same days with continued follow up for 2 years. This was assessed using an IFN- $\gamma$ ELISpot assay according to previously described methods ${ }^{10-12}$. Antigens for the ELISpot assay included three AAV1 capsid peptide pools and the full $\alpha$-SG protein $^{7,11,12}$.

\section{Vector Production}

rAAV1.tMCK.hSGCA was produced at the Harvard Gene Therapy Initiative according to current good manufacturing practices. Vector production followed previously published methods using plasmid DNA tri-transfection of HEK293 cells followed by iodixanol and anion exchange column chromatography purification ${ }^{13}$. The vector was formulated in sterile PBS, and passed all quality control acceptance criteria established by the FDA for strength, identity, and purity.

\section{Efficacy Evaluation}

Efficacy was evaluated by blinded analyses of muscle tissue assessing $\alpha$-SG gene expression by immune stains of muscle sections and western blot analysis with quantification assessed by densitometry with comparisons between sides. This included 4 of 6 blocks in each case, with limitations imposed by spread of vector related to connective tissue barriers in the muscle and by the directional planes of injection. Bioquant image analysis software (Bioquant ${ }^{\circledR}$ Image Analysis Corporation, Nashville, TN) was used for quantitative image analysis. Muscle cross sections $(12 \mu \mathrm{m})$ were subdivided into series of 4 random 10x images. To ensure reliable measurements, staining of the sections and recording of all images were performed during one session with the use of fixed exposure settings and the avoidance of pixel saturation. The lower-intensity threshold was set at background using a LGMD2D section stained with Alexa Fluor 594 goat anti-mouse antibody only, and positive fluorescence was quantified for each section (area percentage). The total $\alpha$-SG fluorescent signal (area percentage) was reported as a ratio to the normal control section which was set at 100 . 
The results of the gene expression findings were presented to our oversight Data Safety Monitoring Board at the National Institutes of Health in a written report before the blind was broken. Major Histocompatability Complex (MHC) I and MHCII antigens (Dako Products) were assessed on muscle sections. CD4+ and CD8+ (BD Biosciences) mononuclear cells were reported as number $/ \mathrm{mm}^{2}$ area. Muscle morphometrics included fiber size histograms.

Statistical analyses were based on differences between the sides in the total number of cells per square millimeter of area expressing CD4+ and CD8+ mononuclear cells, MHCI and MHCII antigens, and muscle fiber size using a paired $t$ test $(p<0.05)$.

\section{Results Study Design}

This was a, double-blind, randomized controlled trial of rAAV1 containing the full length human SGCA under control of the tMCK promoter (rAAV1.tMCK.hSGCA) injected into the EDB muscle of LGMD2D patients ${ }^{7}$. Table 1 summarizes the mutations, age, gender, and disease severity of the current three patients enrolled in cohort 2 (patients $4,5,6$ ) and provides comparative information to Cohort 1 (patients $1,2,3$ previously reported ${ }^{7}$ ). The older age of patients in cohort 2 was incidental to those fulfilling IRB approved criteria for enrollment. The study was approved by the Recombinant DNA Advisory Committee (\# 0610-815; October 31, 2006) and the FDA (IND \# is BB-IND 13434).

Gene transfer was performed in the intensive care unit at Nationwide Children's Hospital. Approximately 4 hours prior to gene transfer, subjects received a dose of intravenous methylprednisolone, $2 \mathrm{mg} / \mathrm{kg}$ (not to exceed $1 \mathrm{gm}$ total). At the time of the procedure investigators received labeled syringes (left and right) on ice containing either viral vector or phosphate buffered saline from the pharmacy. Injection sides were determined by a computer-generated random numbers sequence. Unblinding envelopes were held in the pharmacy. Prior to needle insertion into the muscle the skin was anesthetized with $1 \%$ lidocaine. Gene delivery was guided by ultrasound and electromyographic recordings (37 $\mathrm{mm}$ Teca Myoject injection recording needle) to ensure that muscle was the destination of the delivered product. The picture of the gene delivery to EDB is shown in the report of cohort $1^{7}$. Following injection, the empty syringes were resealed and stored at $-20 \mathrm{C}$. Repeat doses of methylprednisolone were given again at 24 and 48 hours post injection as an anti-inflammatory agent. We have unpublished data that supports the use of glucocorticoids to enhance gene expression both in mouse and nonhuman primate.

EDB muscles were removed bilaterally from patients at 6 months (Table 1 shows exact times of gene transfer and muscle biopsy) and processed for transgene expression and immune reaction to vector and newly expressed protein $^{12}$.

\section{Safety and Efficacy}

Patients were monitored in the hospital for the first 24 hours post gene transfer including hourly vital signs for four hours and then every four hours until discharge. Outpatient evaluations continued and included days 1,2, 7, 14,30,60,120,180, at the end of years 1 and 2 (after the first month outpatient visits could vary by up to 7 days). Photographs of the injection sites were taken immediately and 8 to 12 hours post gene transfer and at each follow up visit. Patients complained of varying degrees of pain post EDB muscle biopsies that required narcotic analgesics for the first night post muscle biopsy. The degree of pain described by the three patients in Cohort 2 was unanticipated based on experience in the prior cohort but should be noted by others performing the procedure. 
No serious adverse events were encountered in any subject. Two had sore throats ( 3 weeks and 9 months post gene transfer), one positive for streptococcal A treated with azithromycin by a primary care physician. A nagging concern in subject 6 was an elevated WBC with normal differential that reached $21.7 \mathrm{~K} / \mathrm{cu} \mathrm{mm}$ on day 2 post gene transfer, day $7(19.2 \mathrm{~K} / \mathrm{cu}$ $\mathrm{mm})$ and day $13(20.1 \mathrm{~K} / \mathrm{cu} \mathrm{mm})$. It then remained in normal range $(<12.5 \mathrm{~K} / \mathrm{cu} \mathrm{mm})$ and suddenly increased transiently on day $200(22.4 \mathrm{~K} / \mathrm{cu} \mathrm{mm})$. The patient was repeatedly checked for respiratory and urinary tract infections, lymphadenopathy, and organomegaly, and no cause was ever found. The AAV capsid T cell response seen by the IFN- $\gamma$ ELISpot assay (presented below) was the only finding that seemed to correlate with the elevated WBCs.

\section{Gene Expression}

Figure 1 shows findings of post gene transfer muscle tissue by immune stains and western blots. Patient 4 (subject 1 in cohort 2) had the mildest phenotype of all six patients receiving gene transfer. $\alpha$-SG expression was seen in the EDB on both sides but gene expression increased by 2 fold on the side of gene transfer (right) as assessed by two separate methods, Bioquant $®$ Image Analysis and western blots. Gene expression reached wild-type levels on the side of gene transfer. We also found an increase in mean muscle fiber diameter comparing control to gene transfer side (untreated $28.2 \pm 11.1$ to treated $52.2 \pm 13.1$ ). The side of gene transfer was validated by quantitative PCR using vector specific primer probes that amplified a unique 5' untranslated leader sequence of the $\alpha$-SG cassette on the right side only. hSGCA transgene copies increased by an average of 150 -fold over baseline (representing 0.06 copies per nucleus) comparing the side of increased gene expression to the contralateral side. The RNase P gene was used as an internal control to normalize for genomic input and confirm the absence of PCR inhibitors in the sample DNA.

Patient 5 was wheelchair-dependent because of severe generalized weakness. There was little residual skeletal muscle $\alpha$-SG providing clear differentiation between control side treated with saline and the side of gene transfer (left). At six months post gene transfer, $\alpha$ SG levels reached wild-type levels accompanied by full restoration of the sarcoglycan complex (Figure 1). Muscle fibers ranged in size from $10 \mu \mathrm{m}$ to $50 \mu \mathrm{m}$ without clear difference between the sides. Quantitative PCR using an $\alpha$-SG specific primer set validated gene transfer to the left EDB. hSGCA transgene copies increased by an average of 1000-fold over baseline (representing 0.64 copies per nucleus) comparing the side of increased gene expression to the contralateral side.

Patient 6 was the exception compared to all previous 5 patients participating in this clinical gene therapy trial. In this case, the six month EDB muscle biopsies showed low level gene expression on both sides and the side of gene transfer (left) could not be differentiated by Bioquant Image analysis or western blots (Figure 1). There was also a very striking paucity of transgene copy numbers per nucleus that was 30 fold lower compared to patient 5 and more than 3 -fold lower compared to patient 4 ( 0.02 copies per nucleus). A one-time muscle biopsy makes it difficult to differentiate between loss of gene expression versus poor muscle transduction at the time of gene transfer. One distinctive feature in this muscle biopsy is the lack of expression of major histocompatability complex I (MHC I) antigen on any muscle fiber in the biopsy. This is in direct contrast to the findings in Patients 4 and 5 of this cohort (Figure 2), as well as MHCI expression on the sarcolemma from patients in the previous cohort $^{7}$. The patient was found to have both early humoral and T-cell responses to AAV1 capsid. The INF- $\gamma$ ELISpot assay demonstrated T cell activation to AAV1 capsid as early as day 2 following gene transfer (also present on day 7) (Figure 3). This is in clear distinction to every other patient undergoing gene transfer in this trial where responses, if present, were not seen until day 14 , as seen in patient 4 of this cohort and in the first three patients in cohort $1^{7}$. $\alpha$-SG antigen specific T cell responses as measured in IFN- $\gamma$ ELISpot assay were 
not observed in PBMCs (Figure 3), distinct from our experience in transferring rAAV.minidystrophin ${ }^{8}$. Accompanying the AAV capsid induced T cell response in patient 6 , we found a very rapid rise in AAV neutralizing antibody titers reaching levels more than 30-times greater than seen in other cases (Figure 4, Table 2). Collectively these findings favor an amnestic response related to pre-existing immunity to AAV. Also of interest, the very rapid immune response in both humoral and $\mathrm{T}$ cell immunity was temporally related to the enigmatic elevations of the peripheral blood WBCs that were increased in the first week post gene transfer and in other weeks that followed.

Recognizing that pre-existing AAV immunity uncovered in patient 6 can present challenges, it was surprising that the neutralizing antibody titer (1:1600) pre-treatment was only 2-fold higher compared to patient 4 (Table 2). The AAV Binding ELISA ${ }^{18}$ was utilized in an attempt to corroborate the cell based AAV1 neutralization data, and revealed a robust particle binding titer of 1:200 in only patient 6 (Table 3), while patient 4 and 5 titers were at baseline $(<1: 50)$.

A final point of interest is that inflammatory cells were found in the post gene transfer muscle biopsies in patients 4,5 , and 6 with differences between cases. CD4+ cells were increased on the side of gene transfer in patients 4 and 5 and decreased in patient 6 [Patient 4: $24.8 \pm 8.6$ vs. $9.5 \pm 4.6$ cells $/ \mathrm{mm}^{2}$, Patient $5: 50.8 \pm 29.5$ vs. $13.2 \pm 5.9$ cells $/ \mathrm{mm}^{2}$, Patient 6: $7 \pm 3.1$ vs. $15.5 \pm 5.1 \mathrm{cells} / \mathrm{mm}^{2}(\mathrm{p}<0.05)$ ], while CD8+ cell counts only reached significance on the side of gene transfer in patient 4 [20.4 \pm 9.6 vs. $0.7 \pm 0.2(p<0.05)]$. Most of the inflammatory infiltrate was focal, perivascular, and in the perimysial connective tissue, at sites distant from individual muscle fibers (Figure 5). We did not encounter invasion of transduced muscle fibers as seen in T cell mediated inflammatory myopathies ${ }^{14}$, suggesting that recruited cells were ineffectual. In the report of the first three subjects undergoing gene transfer with rAAV1.tMCK.hSGCA we showed that focal cell infiltrates expressed TUNEL and interpreted this to imply that recruited inflammatory cells were undergoing programmed cell death ${ }^{7}$. In this second cohort, many of the infiltrating cells in Subjects 4 and 5 were both TUNEL and caspase-3-positive (Figure 5) supporting a similar apoptotic fate ${ }^{15}$.

\section{Discussion}

Gene transfer of rAAV1.tMCK demonstrated persistent gene expression in two of three patients for as long as 6 months. The findings continue to demonstrate the potential merit of AAV1 for protocols employing direct muscle injection ${ }^{16,17}$. The cumulative observations from two successive gene therapy trials using identical conditions of transfer of the hSGCA gene demonstrated robust gene expression in subjects undergoing muscle biopsy at 6 weeks $(\mathrm{n}=2), 3$ months $(\mathrm{n}=1)$, and 6 months $(\mathrm{n}=2)$. These findings appear to address the fundamental question of whether the vector (rAAV1), transgene (hSGCA) and promoter (tMCK) are adequate for further study and can provide the necessary elements to maintain long-term gene expression. The results from this study are encouraging in this regard. Two patients with long-term gene expression showed definite increase in muscle fiber size accompanying persistent gene expression for 3 months (Cohort 1) ${ }^{7}$ and 6 months (Cohort 2). The other patient with very good gene expression for 6 months (Cohort 2 subject 5) was severely affected and we could not discern differences between the side receiving vector and the placebo side. The severity of the dystrophic muscle damage may have impeded change. Additional experience will be required to address potential restoration in severe phenotypes.

The overall experience from this trial is different from our immunological findings related to two scenarios observed in the Duchenne muscular dystrophy (DMD) gene therapy trial ${ }^{13}$. In one DMD case we detected a $\mathrm{T}$ cell response in the ELISpot assay directed against an amino 
acid sequence present in the mini-dystrophin gene but absent from a deleted region of the patient's endogenous dystrophin gene. This situation is unlikely to be encountered in the LGMD2D population, an autosomal recessive disease requiring mutations on both alleles to produce a clinical phenotype. Since missense mutations predominate in LGMD, even a heterozygous deletion mutation at one allele as seen in patient 5 , is not likely to predispose to an immune response from transgene expression. In the DMD trial, an immune reaction was also induced by a novel epitope expressed on revertant muscle fibers that are usually not encountered in LGMD2D patients. On the other hand, we did find that pre-existing immunity to AAV precluded gene expression in this current LGMD2D clinical gene therapy trial. In this case, a rapid amnestic response to AAV was documented (both B and T cells) and predicted an immune-mediated response that correlated with a lack of significant $\alpha$-SG expression in the muscle biopsy taken at the 6-month time point. Absent MHCI expression on muscle fibers and the extremely low vector genome levels in the muscle in the post gene transfer biopsy support this argument. It is difficult to discern, however, if the loss of transduced muscle fibers was caused exclusively through cell-mediated immunity versus a possible role for neutralizing or blocking antibodies hindering initial muscle fiber transduction. The importance of the finding of pre-existing immunity to AAV is that it demonstrates the potential need for cautious selection of patients enrolled in gene transfer trials, especially those not using immunomodulatory therapies. T cell responses directed against AAV disrupting gene expression has previously been reported in a clinical trial gene transfer of Factor IX. ${ }^{18}$ The observation in the current trial that the AAV Binding ELISA appeared to identify the patient with pre-existing immunity to rAAV may indicate a potential role for this laboratory assay in patient selection for gene therapy trials.

The overall favorable findings in this clinical trial lay the foundation for further gene therapy steps that can be taken for LGMD2D patients, a patient group with few treatment options. Persistent gene expression without adverse effects speaks to the safety of gene transfer to muscle and the application of the tMCK promoter that has not previously been used in skeletal muscle gene therapy protocol. The study also opens the door for potential safe and effective delivery of the sarcoglycan gene through the circulation using a protocol similar to the one we have employed in non-human primates permitting vascular delivery to specific muscle groups in the lower limbs ${ }^{19}$.

\section{Acknowledgments}

Yuuki Kaminoh helped characterize the gene mutations in participating patients. Brent Yetter made important contributions to data entry. William M. Fountain IV was responsible for stability assays on the vector; Richard C. Mulligan, Ph.D. and Jeng-Shin Lee, M.D., Ph.D. produced the clinical grade AAV vector at the Harvard Gene Therapy Initiative, Department of Genetics, Harvard Medical School; Xiao Xiao, Ph.D., Department of Pharmacology, University of North Carolina kindly provided the truncated MCK promoter; Kevin P. Campbell, Ph.D., University of Iowa, originally cloned the $50 \mathrm{kDa}$ SGCA used for the toxicology study to obtain the IND and used in the gene construct delivered to subjects in the clinical trial.

This work was supported by the National Institute of Arthritis, Musculoskeletal, and Skin Diseases, National Institutes of Health 1U54NS055958 (JRM) and the Muscular Dystrophy Association, and performed under the FDA IND \# is BB-IND 13434 (JRM). Jesse's Journey (JRM) also provided support.

\section{References}

1. Herzog RW, Cao O, Srivastava A. Two decades of clinical gene therapy--success is finally mounting. Discov Med. 2010; 9:105-11. [PubMed: 20193635]

2. Laval SH, Bushby KM. Limb-girdle muscular dystrophies-from genetics to molecular pathology. Neuropathol Appl Neurobiol. 2004; 30:91-105. [PubMed: 15043707]

3. Wang B, Li J, Fu FH, et al. Construction and analysis of compact muscle-specific promoters for AAV vectors. Gene Ther. 2008; 15:1489-99. [PubMed: 18563184] 
4. Hauser MA, Robinson A, Hartigan-O'Conner D, et al. Analysis of muscle creatine kinase regulatory elements in recombinant adenoviral vectors. Mol Ther. 2000; 2:16-25. [PubMed: 10899824]

5. Carrie A, Piccolo F, Leturcq F. Mutational diversity and hot spots in the alpha-sarcoglycan gene in autosomal recessive muscular dystrophy (LGMD2D). J Med Genet. 1997; 34:470-475. [PubMed: 9192266]

6. Bönnemann CG, Finkel RS. Sarcolemmal proteins and the spectrum of limb-girdle muscular dystrophies. Semin Pediatr Neuro. 2002; 9:81-99.

7. Mendell JR, Rodino-Klapac L, Rosales-Quintero X, et al. Limb girdle muscular dystrophy type 2D gene therapy restores alpha-sarcoglycan and associated proteins. Ann Neurol. 2009; 66:290-297. [PubMed: 19798725]

8. Reese, MG. Application of a time-delay neural network to promoter annotation in the Drosophila melanogaster genome. Comput Chem. 2001; 26:51-6. [PubMed: 11765852]

9. Johnson PR, Schnepp BC, Connell MJ, et al. Novel Adeno associated virus vector vaccine restricts replication of simian immunodeficiency virus in macaques. J Virol. 2005; 79:955-965. [PubMed: 15613324]

10. Chen C-L, Jensen RL, Schnepp BC, et al. Molecular Characterization of Adeno-associated Viruses Infecting Children. J Virol. 2005; 79:14781-14792. [PubMed: 16282478]

11. Manno CS, Pierce GF, Arruda VR, et al. Successful transduction of liver in hemophilia by AAVFactor IX and limitations imposed by the host immune response. Nat Med. 2006; 12:342-347. [PubMed: 16474400]

12. Mendell JR, Campbell K, Rodino-Klapac L, et al. Dystrophin Immunity Revealed by Gene Therapy in Duchenne Muscular Dystrophy. N Engl J Med. 2010 In Press.

13. Rabinowitz JE, Rolling F, Li C, et al. Cross-packaging of a single adeno-associated virus (AAV) type 2 vector genome into multiple AAV serotypes enables transduction with broad specificity. J Virol. 2002; 76:791-801. [PubMed: 11752169]

14. Chahin N, Engel AG. Correlation of muscle biopsy, clinical course, and outcome in PM and sporadic IBM. Neurology. 2008; 70:418-424. [PubMed: 17881720]

15. Wylie AH. "Where, O Death, Is Thy Sting?" A Brief Review of Apoptosis Biology. Mol Neurobiol. Jun 16.2010 Epub.

16. Pacak CA, Conlon T, Mah CS, Byrne BJ. Relative persistence of AAV serotype 1 vector genomes in dystrophic muscle. Genet Vaccines Ther. 2008; 6:14. [PubMed: 18854054]

17. Pacak CA, Walter GA, Gaidosh G, et al. Long-term skeletal muscle protection after gene transfer in a mouse model of LGMD-2D. Mol Ther. 2007; 15:1775-81. [PubMed: 17653106]

18. Manno CS, Pierce GF, Arruda VR, et al. Successful transduction of liver in hemophilia by AAVFactor IX and limitations imposed by the host immune response. Nat Med. 2006; 12:342-347. [PubMed: 16474400]

19. Rodino-Klapac LR, Montgomery CL, Bremer WG, Malik V, Shontz KM, Davis N, Sprinkle S, Campbell KJ, Sahenk Z, Clark KR, Walker CM, Mendell JR, Chicoine LG. Persistent expression of FLAG tagged micro-dystrophin in non-human primates with intramuscular and vascular approaches. Mol Ther. 2010; 18:109-17. Epub 2Nov 10ePUB Nov 2009. [PubMed: 19904237] 
a
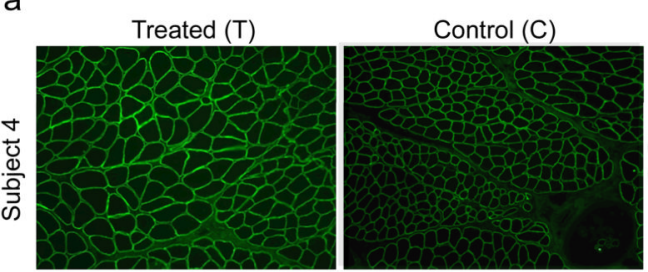

b

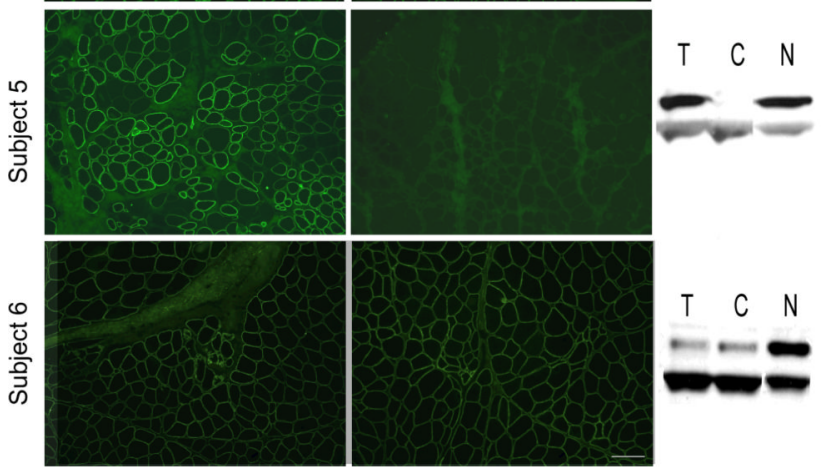

c

Beta-Sarcoglycan

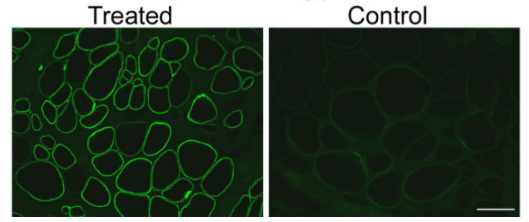

Figure 1.

a). Subjects 4-6: post gene transfer tissue sections from EDB muscles were stained with antibody to $\alpha$-SG. Subjects 4 and 5 showed increased staining on treated side (T) compared to control side (C). Subject 6 showed no difference in $\alpha$-SG staining intensity pre- and postgene transfer (findings verified by Bioquant Image Analysis $®$ ) (scale bar $=150 \mu \mathrm{m})$. b). Subjects 4-6: western blots show increased $\alpha$-SG gene expression on the side of gene transfer compared to the contralateral side (treated on left, control on right) for Subjects 4 and 5; residual gene expression from mutant protein prominently exhibited in Subject 4. Subject 6 showed no increase in $\alpha$-SG expression comparing pre- and post-gene transfer gels. WBs normalized to actin (lower band) and each is compared with normal (N) muscle for comparison. c). Subject 5: $\beta$-sarcoglycan staining (Subject 5) demonstrates restoration on the side of gene transfer with absent staining on control side (scale bar $=100 \mu \mathrm{m})$. Other sarcoglycans (delta and gamma) were also restored (not shown). 

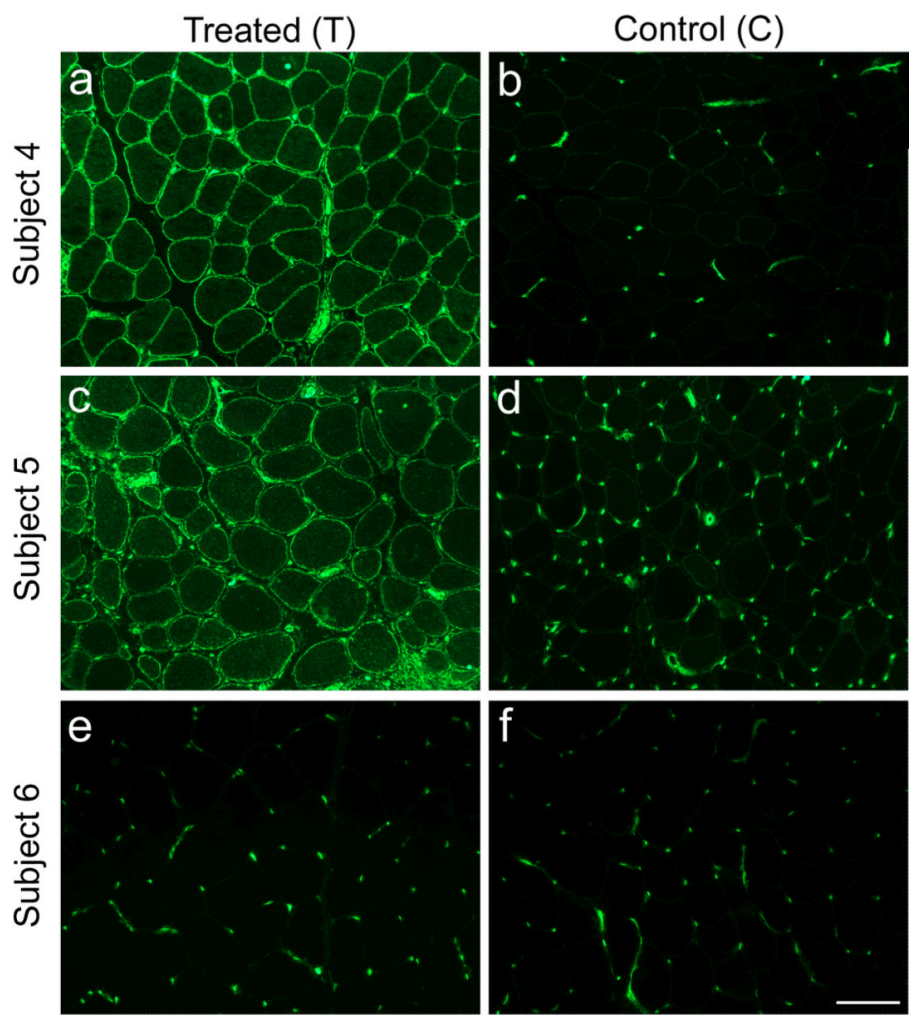

Figure 2.

MHCI staining of sarcolemmal membrane of muscle sections on treated (T) and control (C) sides. Subjects 4 and 5 show staining on the treated $(a, c)$ but not the control (b, d) sides. Subject 6 shows no MHCI staining on treated side (e) or control side (f). (scale bar $=100$ $\mu \mathrm{m})$. Microvascular circulation is positive for MHC I on both sides in all subjects. 

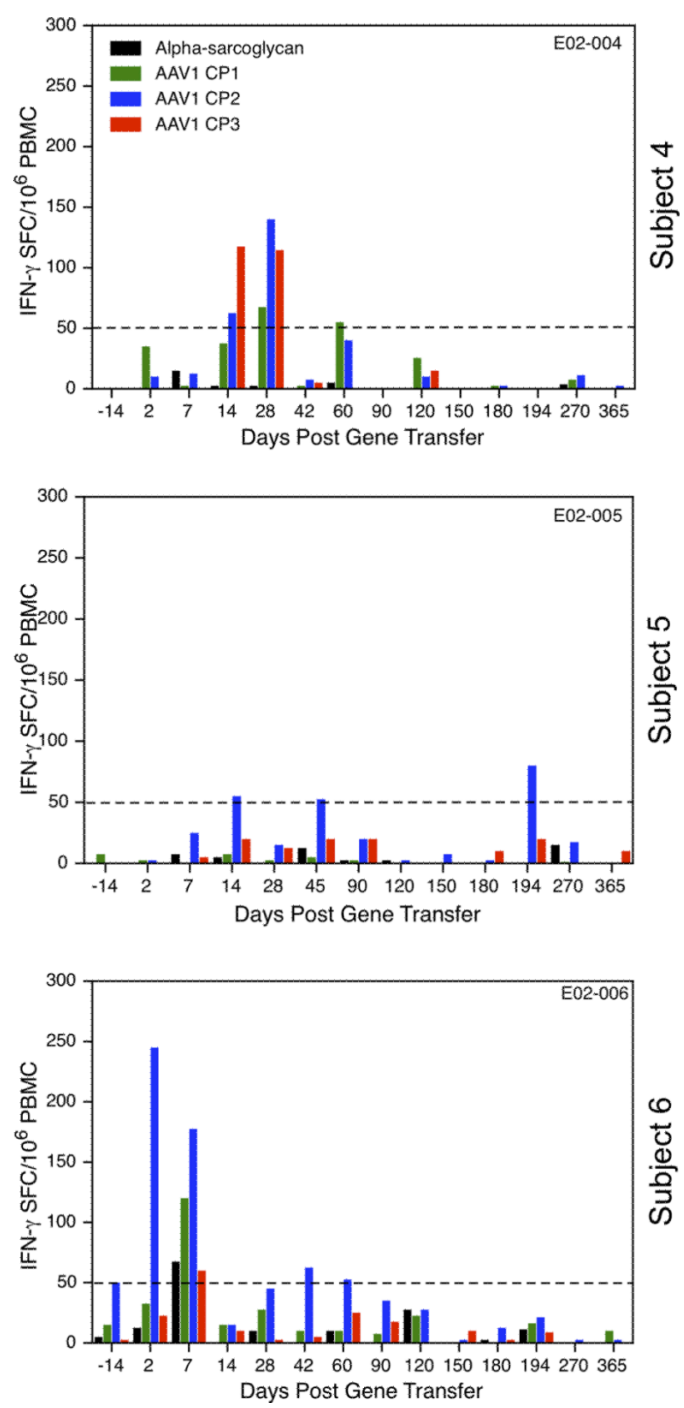

Figure 3.

a). Subject 4 (EO2-004): $\alpha-S G$ stimulated IFN- $\gamma$ ELISpot assays showed no increase in spot forming colonies (SFC) per million peripheral blood mononuclear cells (PBMCs)at any time point (prior to gene transfer -14 days up to day 365). AAV1 capsid peptide pool stimulation (CP2 blue and CP3 red) were positive for $\mathrm{T}$ cell response (exceeded confidence limits of $>50$ spot forming cells /million PBMCs) at days 14 and 28 . The response to AAV1 capsid pool 1 (CP1 green) was minimally positive at day 14. b). Subject 5 (E02-005):): $\alpha$ SG and AAV1 capsid INF- $\gamma$ ELISpot assays were negative preceding and throughout the course of gene transfer up to day 365 with exception of a slight reaction at day 194 to AAV1 capsid pool 2. c). Subject 6 (E02-006): the $\alpha$-SG stimulated IFN- $\gamma$ ELISpot assays (black) were consistently negative pre- and post-gene transfer. However, this patient showed a prolific AAV1 capsid response as early as day 2 and also present on day 7 . This was distinctly earlier than other patients in Cohort 2 or in Cohort 1 (previously published ${ }^{7}$ ). 


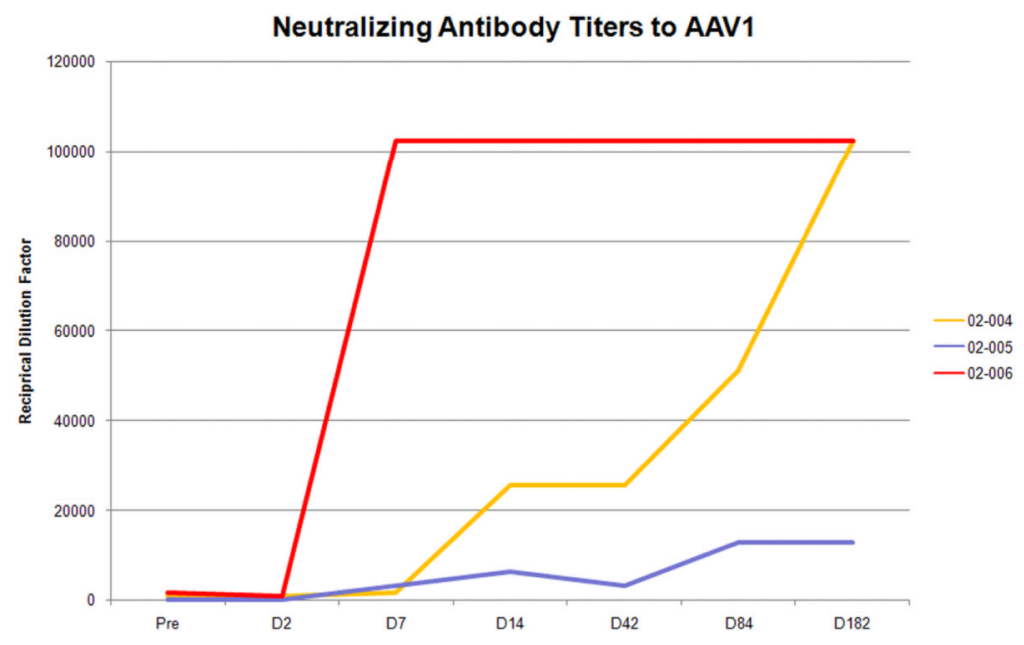

Figure 4.

Neutralizing antibody titers to AAV1 on Subjects 4-6 starting pre (day -14) through day 182 (D182) post-gene transfer. In all three subjects serum neutralizing antibody titers to AAV1 were very low (see Table 2 for pre-treatment titers). Follow up studies showed a slow rise in serum AAV1 titers in Subjects 4 and 5. This contrasted with the rapid rise and peak elevation by week 1 in Subject 6. Plateau in elevation for Subject 6 (red) represents limit of assay. 

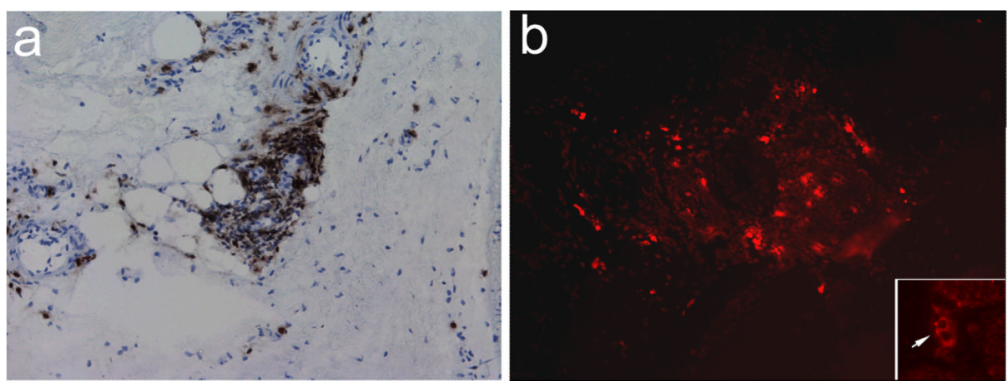

Figure 5.

a) Subject 4. Focal collection of CD8+ T cells shown in perimysial connective tissue of muscle biopsy post gene transfer. b). Subject 4. TUNEL staining demonstrates inflammatory cells undergoing apoptosis confirmed by anti-caspase 3 antibody (inset). 


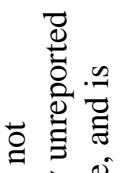

空 $\frac{0}{0}$

달

I D

苞泀离

需

$\dot{\overrightarrow{0}} \Xi$

离

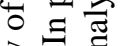

各官

.

可

造造范

害

8 更

प्रे है

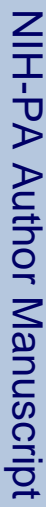

造

횽

$-\quad \bar{d}$

응

齐当

ते $\frac{7}{0}$

总言艺

氙

$\$$ \&

I音

跣:

. 흉

त 융

记氙

¿ 08

के

का एँ

$\Xi \stackrel{\square}{\mathscr{D}}$

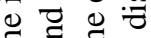

踏

उ㝴

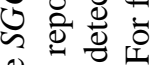

류

$\Xi 0 \quad:=$

ปี

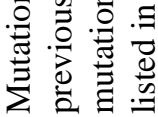

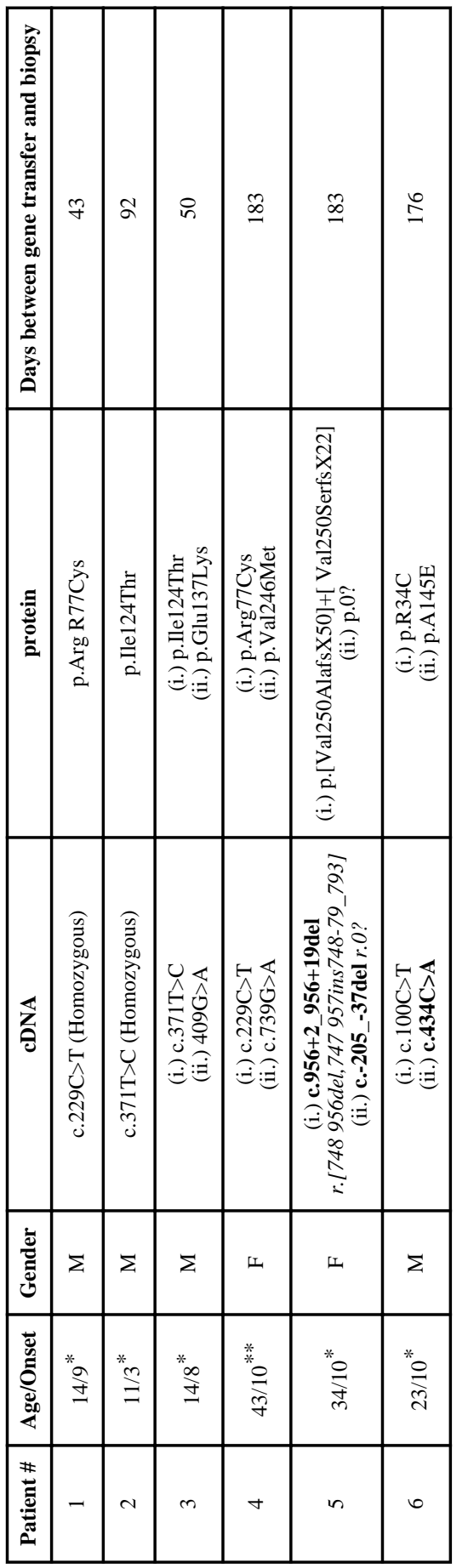

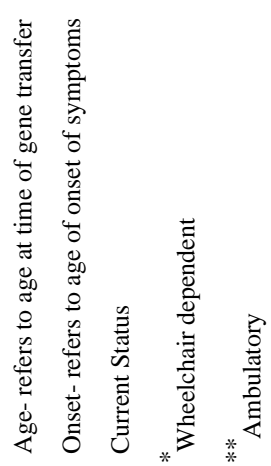


Table 2

\section{Neutralizing antibody titers against AAV1 pre and post-gene transfer}

ELISA assay for neutralizing antibody titers against AAV1 pre- and post-gene transfer. The results for Subject 6 are one-dilution higher pre-treatment compared to subject 4 . However, by day 7 the neutralizing antibody titers rose $>30$-fold compared to other subjects.

\begin{tabular}{|c|c|c|c|}
\hline Time Post Injection & Patient \#4 (E02-004) & Patient \#5 (E02-005) & Patient \#6 E02-006 \\
\hline Pre-treatment & $1: 800$ & $<1: 50$ & $1: 1600$ \\
\hline Day 7 & $1: 1600$ & $1: 3200$ & $>1: 102400$ \\
\hline 2 weeks & $1: 25600$ & $1: 6400$ & $>1: 102400$ \\
\hline 6 weeks & $1: 25600$ & $1: 3200$ & $>1: 102400$ \\
\hline 12 weeks & $1: 51200$ & $1: 12800$ & $>1: 102400$ \\
\hline 26 weeks (6 months) & $>1: 102400$ & $1: 12800$ & $>1: 102400$ \\
\hline
\end{tabular}


Table 3

\section{Binding antibody ratios against AAV1 pre-gene transfer}

Binding antibody ratios against AAV1 shows dramatic differences pre-treatment for Subject 6 compared to Subjects 4 and 5. While neutralizing antibody titers (Table 2) failed to correlate with gene expression, the unequivocally higher (>1000 fold higher) binding antibody ratio in patient 6 predicted an amnestic response with early onset humoral and $\mathrm{T}$ cell immunity and poor gene transfer..

\begin{tabular}{|c|c|c|c|}
\hline Dilution & Patient \#4 (E02-004) & Patient \#5 (E02-005) & Patient \#6 E02-006 \\
\hline $1: 50$ & $\mathbf{0 . 0 1 6}$ & $\mathbf{0 . 0 0 6}$ & $\mathbf{1 0 . 1 7 8}$ \\
\hline $1: 100$ & $\mathbf{0 . 0 3 2}$ & -0.045 & $\mathbf{6 . 0 5 9}$ \\
\hline $1: 200$ & $\mathbf{0 . 0 0 3}$ & $\mathbf{0 . 0 1 3}$ & $\mathbf{3 . 3 3 5}$ \\
\hline $1: 400$ & $\mathbf{0 . 0 1 4}$ & -0.001 & $\mathbf{1 . 6 6 6}$ \\
\hline $1: 800$ & $\mathbf{0 . 0 2 9}$ & $\mathbf{0 . 0 0 3}$ & $\mathbf{0 . 8 1 2}$ \\
\hline $1: 1600$ & $\mathbf{0 . 0 1 4}$ & $\mathbf{0 . 0 0 0}$ & $\mathbf{0 . 3 9 7}$ \\
\hline
\end{tabular}

